\title{
Effects of non-standard neutrino interactions on MSW-LMA solution to the solar neutrino problem
}

\author{
M. M. Guzzo * P. C. de Holanda $\dagger$ and O. L. G. Pere $\#$ \\ Instituto de Física Gleb Wataghin - UNICAMP, 13083-970 Campinas SP, Brazil
}

\begin{abstract}
We show that the non-standard neutrino interactions can play a role as sub-leading effect on the solar neutrino oscillations. We observe that very small flavor universality violations of order of 0.1-0.2 $G_{F}$ is sufficient to induce two phenomena: suppression of the $\nu_{e}$-earth regeneration and a shift of the resonance layer in the sun. We obtain these phenomena even in the absence of any flavor changing interactions. We discuss their consequences and confront with a global analysis of solar+KamLAND results. We conclude that a new compatibility region in the $\Delta m^{2} \times \tan ^{2} \theta_{\odot}$, which we call very low Large Mixing Angle region is found for $\Delta m^{2} \sim 10^{-5} \mathrm{eV}^{2}$ and $\tan ^{2} \theta_{\odot}=0.45$.
\end{abstract}

PACS numbers: 14.16.Pq, 28.50.Hw

\section{INTRODUCTION}

In the last years, the discovery of neutrino oscillation in solar and reactor experiments selected as a more probable explanation to the solar neutrino problem the so called Large Mixing Angle (LMA) MSW solution. The SNO [1, 2] and the KamLAND [4] experiments confirm and refine the trend of the evidences of neutrino oscillations due the solar neutrino observations, as measured by Homestake [5], SAGE [6], GALLEX [7], GNO [] and Super-Kamiokande [9, 10]. As a result, the solar oscillation parameters have pinned down to $6 \times 10^{-5} \mathrm{eV}^{2}<\Delta m^{2}<1 \times 10^{-4} \mathrm{eV}^{2}$ and $0.3<\tan ^{2} \theta_{\odot}<0.55$ at $2 \sigma$ [1]. Several analyzes have arrived to same conclusions [12, 13, 14, 15, 16, 17].

In a more general context, sub-leading effects can change this picture, which motivate us to investigate the robustness of the determination of the solar parameters. In this letter, we assume that non-standard neutrino interactions, which we parameterized by two parameters $\epsilon^{\prime}$ and $\epsilon$, are present, relaxing the allowed region of the parameters. In the presence of non-standard neutrino interactions, we have found that the allowed interval for $\Delta m^{2}$ increases, rescuing the very low LMA region, $\Delta m^{2} \sim 1 \times 10^{-5} \mathrm{eV}^{2}$, and the high part of LMA region, $\Delta m^{2} \sim 2 \times 10^{-4} \mathrm{eV}^{2}$, respectively due the suppression of earth matter and due to a $\Delta m^{2}$ shift induced by a non-zero $\epsilon^{\prime}$.

\section{NEUTRINO EVOLUTION: MSW MECHANISM AND NON-STANDARD NEUTRINO INTERACTIONS}

We will work in a two generation neutrino scheme, with the contribution from the non-standard neutrino interactions (NSNI) 18, 19, 20] added to the usual MSW Hamiltonian 21]. The Hamiltonian in the flavor basis equals

$$
H=H_{M S W}+H_{N S N I}
$$

*Electronic address: guzzo@ifi.unicamp.br

${ }^{\dagger}$ Electronic address: holanda@ifi.unicamp.br

‡Electronic address: orlando@ifi.unicamp.br 
where

$$
H_{M S W}=\left[\begin{array}{cc}
+\sqrt{2} G_{F} N_{e}(r)-\frac{\Delta m^{2}}{4 E} \cos 2 \theta & \frac{\Delta m^{2}}{4 E^{2}} \sin 2 \theta \\
\frac{\Delta m^{2}}{4 E} \sin 2 \theta & \frac{\Delta m^{2}}{4 E} \cos 2 \theta
\end{array}\right]
$$

and

$$
H_{N S N I}=\left[\begin{array}{cc}
0 & \sqrt{2} G_{F} \epsilon_{f} N_{f}(r) \\
\sqrt{2} G_{F} \epsilon_{f} N_{f}(r) & \sqrt{2} G_{F} \epsilon_{f}^{\prime} N_{f}(r)
\end{array}\right]
$$

where $N_{f}=N_{e}+2 N_{n}$ when the NSNI occur with d-quarks, $N_{f}=2 N_{e}+N_{n}$ when u-quarks are involved and simply $N_{f}=N_{e}$ when we have electrons. The parameters $\epsilon$ and $\epsilon^{\prime}$ describe, respectively, the relative strength of the flavor changing neutrino interactions and the new flavor diagonal, but non-universal interactions, normalized to $G_{F}$.

The NSNI parameters are constrained by non-universal and flavor-changing processes to be $\epsilon^{\prime}<0.7$ and $\epsilon<$ $10^{-2}$ 20]. Since we concentrate on the regions around LMA neutrino parameters, effects of $\epsilon$ parameters will be negligibly small and we can, effectively, set this parameter to zero. We solve numerically the evolution equation, using the density profile of the Sun 22] or the earth [23].

We now discuss the behaviour of $\nu_{e}$-survival probability that will help to understand our results. In the usual MSW mechanism, for the solar parameters in the LMA region, we have a resonant behaviour inside the sun given by

$$
\left(\frac{\Delta m^{2}}{4 E} \cos 2 \theta\right)\left(\frac{2}{V_{0}}\right) \equiv \frac{\Delta m^{2} \cos 2 \theta}{2 \sqrt{2} E G_{F} N_{e}} \sim 1
$$

In the Sun, $N_{n} \sim[0.1-0.3] N_{e}$, and a positive value of $\epsilon^{\prime}$ can be interpreted as a small negative correction in the solar density. For a given $\Delta m^{2}$ and $\theta$, the resonance is displaced to the center of the Sun, and as a consequence, less neutrinos experience the resonance. As a result, the transition between resonant and non-resonant survival probability is displaced to higher values of $\Delta m^{2} / 4 E$, according to:

$$
\frac{\Delta m^{2}}{4 E} \rightarrow \frac{\Delta m^{2}}{4 E}-\frac{1}{\cos 2 \theta} \frac{1}{\sqrt{2}} G_{F} \epsilon_{f}^{\prime} N_{f}(r)
$$

As stated above, effects of a non-vanishing $\epsilon$ are much weaker, since for LMA we have $\Delta m^{2} /(4 E) \sim 10^{-12} \mathrm{eV}$, the same order of $\sqrt{2} G_{F} N_{f}(r)$ at the resonance region in the sun. Therefore we should have $\epsilon \sim 1$, much above the experimental limit, to have some effect on the survival probability. We neglect $\epsilon$ in what follows.

In the earth $N_{e}=N_{p} \sim N_{n} \sim 1 / 3 N_{d}$. Therefore, for values of $\epsilon^{\prime} \sim 1 / 3$ the matter term in the evolution matrix due to NSNI has the same order of magnitude of the ordinary matter term. As a consequence, the regeneration of $\nu_{e}$ is suppressed.

Both these effects are presented in Fig. [1 Around $\Delta m^{2} / 4 E \sim\left[10^{-12}-10^{-11}\right] \mathrm{eV}$ we can see the displacement in $\Delta m^{2} / 4 E$ of the survival probability in the Sun, and around $\Delta m^{2} / 4 E \sim\left[10^{-14}-10^{-12}\right] \mathrm{eV}^{2}$ the suppression in the regeneration effect is effective. 


\section{SOLAR NEUTRINO AND KAMLAND DATA ANALYSIS}

We use for the solar neutrino analysis the same data set and the same procedure of analysis appearing in Ref. [11]. Here the main ingredients of the analysis are summarized.

The data sample consists of

- 3 total rates: (i) the $A r$-production rate, $Q_{A r}$, from Homestake [5], (ii) the $G e$-production rate, $Q_{G e}$ from SAGE

6] and (iii) the combined $G e$-production rate from GALLEX and GNO 8];

- 44 data points from the zenith-spectra measured by Super-Kamiokande during 1496 days of operation [9];

- 34 day-night spectral points from SNO plus CC, NC and ES rates from SNO salt-phase [2];

- 3 fluxes from the SNO salt phase [3] measured by the CC-, NC and ES- reactions.

Altogether the solar neutrino experiments provide us with 84 data points. All the solar neutrino fluxes are taken according to SSM BP2000 [24].

Thus, in our analysis of the solar neutrino data we have three fit parameters: $\Delta m^{2}, \tan ^{2} \theta_{\odot}$ and $\epsilon^{\prime}$.

We define the contribution of the solar neutrino data to $\chi^{2}$ as

$$
\chi_{\text {sun }}^{2}=\sum_{i, j=1,84}\left(R_{t h}^{i}-R_{e x}^{i}\right) \sigma_{i, j}^{-2}\left(R_{t h}^{j}-R_{e x}^{j}\right)
$$

where we construct the $84 \times 84$ covariance matrix $\sigma_{i, j}^{2}$ taking in consideration all correlations between uncertainties.

Following the procedure done in Ref. 25], the KamLAND data are analyzed through a Poisson statistics, using the following $\chi^{2}$ :

$$
\chi_{K L}^{2}=\sum_{i=1,13} 2\left[N_{i}^{t h}-N_{i}^{o b s}+N_{i}^{o b s} \ln \left(\frac{N_{i}^{o b s}}{N_{i}^{t h}}\right)\right],
$$

where the $l n$ term is absent when bins with no events are considered ( 5 last bins).

The combined analysis of solar + KamLAND data is done just adding the two contributions in $\chi^{2}$ :

$$
\chi^{2}=\chi_{K L}^{2}+\chi_{\text {sun }}^{2}
$$

We minimize the global $\chi^{2}$ with respect to the three parameters $\Delta m^{2}, \tan ^{2} \theta_{\odot}$ and $\epsilon^{\prime}$. For the KamLAND $\chi_{K L}^{2}$, the effect of $\epsilon^{\prime}$ is negligible due to the short distance traveled inside earth, then effectively $\chi_{K L}^{2}$ depends only on $\Delta m^{2}$ and $\tan ^{2} \theta_{\odot}$. We show our results in the plane $\Delta m^{2}$ and $\tan ^{2} \theta_{\odot}$, in Fig. 2 where we minimized away the dependence on the $\epsilon^{\prime}$ parameter. The best fit is for $\epsilon^{\prime}=0$, and the usual MSW mechanism is still the best solution and any non-zero value for $\epsilon^{\prime}$ parameter only worsens the fit.

To have an idea of the bounds put by the present data and the possible accumulation of more statistics on KamLAND, we plot in the upper panel of Fig. 3 the bounds on $\epsilon^{\prime}$ parameter. The present bound is shown by a thick solid 
curve, where we have $\epsilon_{d}^{\prime}<0.3$ at $2 \sigma$. Assuming a future exposure of 1 kton-yr for KamLAND experiment and the present solar neutrino data, we simulate the KamLAND data as generated by a specific $\Delta m^{2}$ and tan ${ }^{2} \theta_{\odot} \operatorname{combination}$ located in each one of three islands: very low, normal, and high LMA region. If we assume a point in the normal LMA region, we will get after 1 kton-yr for KamLAND, $-0.4<\epsilon_{d}^{\prime}<0.25$ at $2 \sigma$ as showed in the dashed curve. Similar plots for the high (very low) LMA region as long-dashed (dotted) curves show that the bounds will be $\epsilon_{d}^{\prime}<-0.16$ $\left(0.16<\epsilon_{d}^{\prime}<0.35\right)$

\section{A. NSNI with u-quarks and electrons}

All our results showed were computed for a NSNI with d-quarks. In this section we summarized the main differences when you have NSNI with u-quarks and electrons.

To get NSNI with u-quarks, we need to replace $N_{d} \rightarrow N_{u}$ in the evolution equation for neutrinos. In the production region where most of ${ }^{8} B$ neutrinos are created, the ratio between the $N_{d}$ and $N_{u}$ densities is almost constant, as can be seen in Figure 3 of Ref. [20]. Then the conversion probability showed in Fig. 1 for d-quarks, is similar to the conversion induced by u-quarks, with the appropriate rescaling of $\epsilon_{u}^{\prime}$ parameter: $\epsilon_{u}^{\prime}=\epsilon_{d}^{\prime} * N_{d} / N_{u}$. In the earth the differences are minimal. If you compare the allowed region for d-quarks (see Fig. 2 ) and u-quarks then we have practically the same figure for u-quarks.

For NSNI induced by electrons, we can think as a rescaling of the usual matter potential of the MSW mechanism: $\sqrt{2} G_{F} N_{e}(r) \rightarrow \sqrt{2} G_{F} N_{e}(r)-\sqrt{2} G_{F} \epsilon_{e}^{\prime} N_{e}(r)=\sqrt{2}\left(1-\epsilon_{e}^{\prime}\right) G_{F} N_{e}(r)$. The parameter $\epsilon_{e}^{\prime}$ have looser bounds then $\epsilon_{d}^{\prime}$ 27] and values for $\epsilon_{e}^{\prime} \sim 1$ are still allowed. One could worry that such large values of $\epsilon_{e}^{\prime}$ could cause a strong effect on the detection cross section $\sigma(\nu e \rightarrow \nu e)$ used to detect solar neutrinos on Super Kamiokande experiment. This is not true because the matter potential induced by $\epsilon_{e}^{\prime}$ is proportional only to the vector contribution of the non-standard neutrino couplings, and the cross section depends on a combination of the left/right couplings of the non-standard neutrino interaction of the electron neutrino.

When we looked for the allowed regions, we have similar figures as in Fig. 2 and we obtain again the appearance of a very low LMA region. We have also shown in Fig. 3 (second and third panels) the limits in $\epsilon^{\prime}$ for NSNI induced by u-quarks and electrons. For comparison, similar plot was obtained in Ref. [28], bu.

\section{DISCUSSION OF RESULTS}

In the allowed region showed, we notice two distinguished facts: the appearance of new very low LMA region at $\Delta m^{2} \sim 10^{-5} \mathrm{eV}^{2}$ and the old very high LMA, $\Delta m^{2} \sim 1 \times 10^{-4} \mathrm{eV}^{2}$, appears at $1 \sigma$.

The analysis of the data, in the absence of $\epsilon^{\prime}$ parameter, disfavor the high LMA solution that is only allowed at

$3 \sigma$ [1]. Assuming a negative $\epsilon^{\prime}$, the situation changes due the shift of the resonance layer in the sun, as commented before. For $\epsilon^{\prime}=-0.6$ (the lower limit we used in our analysis) this region is now accepted at $1 \sigma$ C.L.. 
The most interesting phenomenology happens at the island of allowed region in KamLAND analysis around $\Delta m^{2} \sim$ $10^{-5} \mathrm{eV}^{2}$. This region is not allowed in a pure-LMA scenario due to the very high day-night asymmetry that is expected for these parameters. But when we include a $\epsilon^{\prime}>0$, the NSNI term in Hamiltonian compensates the effect of Earth matter interaction. For $\epsilon^{\prime} \sim 1 / 3$ we expect a very low regeneration, and for larger values of $\epsilon^{\prime}$ we can have even a positive day-night asymmetry. This region is also allowed when we assume other non-standard mechanisms, as random matter density fluctuations in the sun, as presented in [26].

\section{CONCLUSIONS}

We showed that NSNI will affect the fit in the LMA region of the MSW solution to the solar neutrino anomaly. When one takes into account the KamLAND results, positive values of the $\epsilon^{\prime}$ push the allowed region of the neutrino parameters $\Delta m^{2}$ and $\tan ^{2} \theta_{\odot}$ at $95 \%$ C.L. from pure MSW low-LMA and high-LMA to a completely new region in which $\Delta m^{2}$ is lower than the previous two ones, which we call very-low-LMA. If one chooses $\epsilon^{\prime}<0$, the preferred allowed region tends to higher values of $\Delta m^{2}$.

Almost all our conclusions below are independent of specific sources of the non-standard neutrino interactions, that could be present in interactions with d-quarks, u-quarks or electrons.

We have found that the main effects of the presence of the NSNI interactions are:

- displacement of low-LMA region to lower (higher) values of $\Delta m^{2}$, for a positive (negative) value of $\epsilon^{\prime}$.

- suppression of Earth regeneration at $\Delta m^{2} \sim 10^{-5} \mathrm{eV}^{2}$ for positive values of $\epsilon^{\prime}$.

- Due to suppression of Earth regeneration, appearance of a new region of compatibility between solar and KamLAND data around $\Delta m^{2} \sim 10^{-5} \mathrm{eV}^{2}$, with no spectrum distortion for the low-energy SK and SNO data.

- improvement of high-LMA fit quality for positive values of $\epsilon^{\prime}$

- a 1 kton-yr of KamLAND can make a strong statement about the existence of non-standard neutrino interactions.

The striking signal of this NSNI would be the location of the prefered oscillation parameters in the very low or in the high LMA region.

Note added: When we were finishing our paper, an article by Friedland, Lunardini and Peña-Garay (hep-ph/0402266) appeared, which discusses topics similar to the ones discussed in our paper, where we discuss not only the non-standard neutrino interaction induced by d-quarks case as well the u-quarks and electrons. Also we made a quantitative statement about the role of more statistics on KamLAND experiment, combined with the present solar neutrino data, to put more restrictive bounds on non-standard neutrino interactions. 


\section{Acknowledgments}

This work was supported by Fundação de Amparo à Pesquisa do Estado de São Paulo (FAPESP) and Conselho Nacional de Desenvolvimento Científico e Tecnológico (CNPq).

[1] SNO collaboration, Q. R. Ahmad et al., Phys. Rev. Lett. 87, 071301 (2001).

[2] SNO collaboration, Q. R. Ahmad et al., Phys. Rev. Lett. 89, 011301 (2002); 011302 (2002); nucl-ex/0309004

[3] SNO collaboration, Q. R. Ahmad et al., nucl-ex/0309004

[4] KamLAND collaboration, K. Eguchi et al., Phys. Rev. Lett, 90, 021802 (2003).

[5] Homestake collaboration, B. T. Cleveland et al., Astroph. J. 496, 505 (1998).

[6] SAGE collaboration, J.N. Abdurashitov et al. Zh. Eksp. Teor. Fiz. 122, 211 (2002) [J. Exp. Theor. Phys. 95, 181 (2002)], astro-ph/0204245 V. N. Gavrin, Talk given at the VIIIth International conference on Topics in Astroparticle and Underground Physics (TAUP 03), Seattle, Sept. 5 - 9, 2003.

[7] GALLEX collaboration, W. Hampel et al., Phys. Lett. B 447, 127 (1999).

[8] GNO Collaboration, E. Belotti, Talk given at the VIIIth International conference on Topics in Astroparticle and Underground Physics (TAUP 03), Seattle, Sept. 5 - 9, 2003.

[9] Super-Kamiokande collaboration, S. Fukuda et al., Phys. Rev. Lett. 86, 5651 (2001); Phys. Rev. Lett. 86, 5656 (2001), Phys. Lett. B 539, 179 (2002).

[10] Super-Kamiokande collaboration, M. B. Smy et al., Phys. Rev. D 69, 011104 (2004).

[11] P. C. de Holanda, A. Yu. Smirnov, hep-ph/0309299, to be published at Astropart. Phys..

[12] A. B. Balantekin and H. Yüksel, Phys. Rev. D 68, 113002 (2003).

[13] G. L. Fogli, E. Lisi, A. Marrone, D. Montanino, A. Palazzo and A. M. Rotunno, Phys. Rev. D 67, 073002 (2003); G. L. Fogli, E. Lisi, A. Marrone, D. Montanino, A. Palazzo and A. M. Rotunno, Phys. Rev. D 69, 017301 (2004).

[14] M. Maltoni, T. Schwetz, M. A. Tortola and J.W.F. Valle, Phys. Rev. D 68, 113010 (2003).

[15] P. Aliani, V. Antonelli, M. Picariello and E. Torrente-Lujan, hep-ph/0309156

[16] P. Creminelli, G. Signorelli and A. Strumia, JHEP 0105, 052 (2001); See addendum, hep-ph/0102234 v.5.

[17] A. Bandyopadhyay, S. Choubey, S. Goswami, S. T. Petcov and D.P. Roy, Phys. Lett. B 583, 134 (2004).

[18] L. Wolfenstein, Phys. Rev. D 17, 2369 (1978).

[19] M.M. Guzzo, A. Masiero, S.T. Petcov, Phys. Lett. B 260154 (1991).

[20] S. Bergmann, M. M. Guzzo, P. C. de Holanda, P. I. Krastev and H. Nunokawa, Phys. Rev. D 62, 073001 (2000).

[21] L. Wolfenstein, Phys. Rev. D 17, 2369 (1978); L. Wolfenstein, in "Neutrino-78", Purdue Univ. C3 - C6, (1978); S. P. Mikheyev and A. Yu. Smirnov, Yad. Fiz. 42, 1441 (1985) [ Sov. J. Nucl. Phys. 42, 913 (1985)]; Nuovo Cim. C9, 17 (1986); S. P. Mikheyev and A. Yu. Smirnov, ZHETF, 91, (1986), [Sov. Phys. JETP, 64, 4 (1986)].

[22] J. N. Bahcall, M.H. Pinsonneault and S. Basu, Astrophys. J. 555, 990 (2001).

[23] A. M. Dziewonski and D. L. Anderson, Phys. Earth Planet. Interiors 25 (1981) 297; E. Lisi and D. Montanino, Phys. Rev. D56, 1792 (1997).

[24] J. N. Bahcall, M.H. Pinsonneault and S. Basu, Astrophys. J. 555 (2001) 990.

[25] P. C. de Holanda and A. Y. Smirnov, JCAP 0302, 001 (2003).

[26] M. M. Guzzo, P. C. de Holanda and N. Reggiani, Phys. Lett. B 569, 45 (2003).

[27] M. M. Guzzo, H. Nunokawa, P. C. de Holanda and O. L. G. Peres, Phys. Rev. D 64, 097301 (2001)

[28] G. L. Fogli, E. Lisi, A. Palazzo and A. M. Rotunno, Phys. Rev. D 67, 073001 (2003); G. L. Fogli, E. Lisi, A. Marrone and A. Palazzo, Phys. Lett. B 583, 149 (2004).

[29] A. Friedland, C. Lunardini and C. Pena-Garay, hep-ph/0402266 


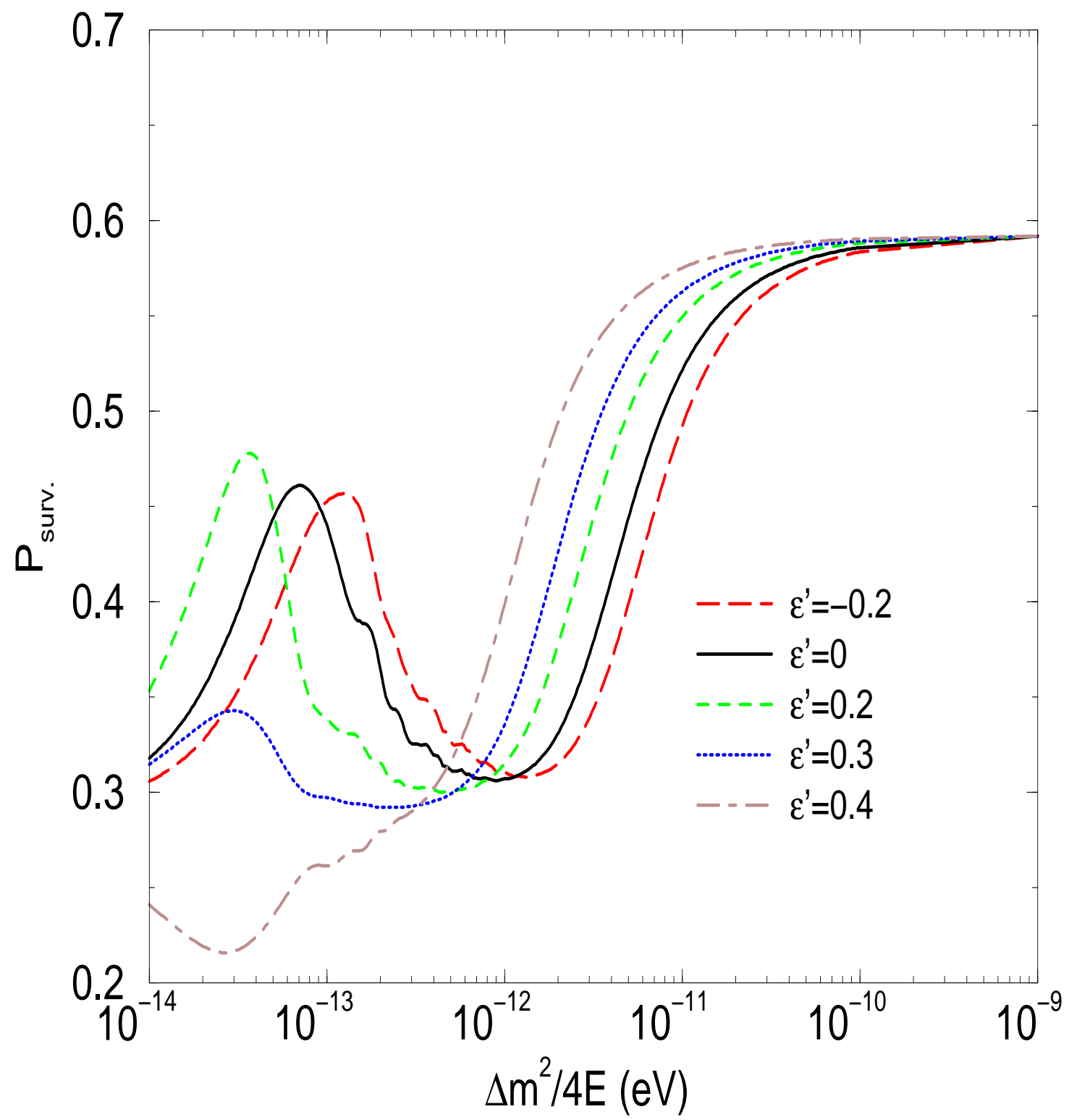

FIG. 1: Survival probability of electron neutrinos. In this figure we can see the displacement in $\Delta m^{2} / 4 E$ of the suppression pit associated with the transition between resonant and non-resonant regions. Also is possible to see the supression of regeneration effect for $\epsilon^{\prime} \sim 0.3$. 


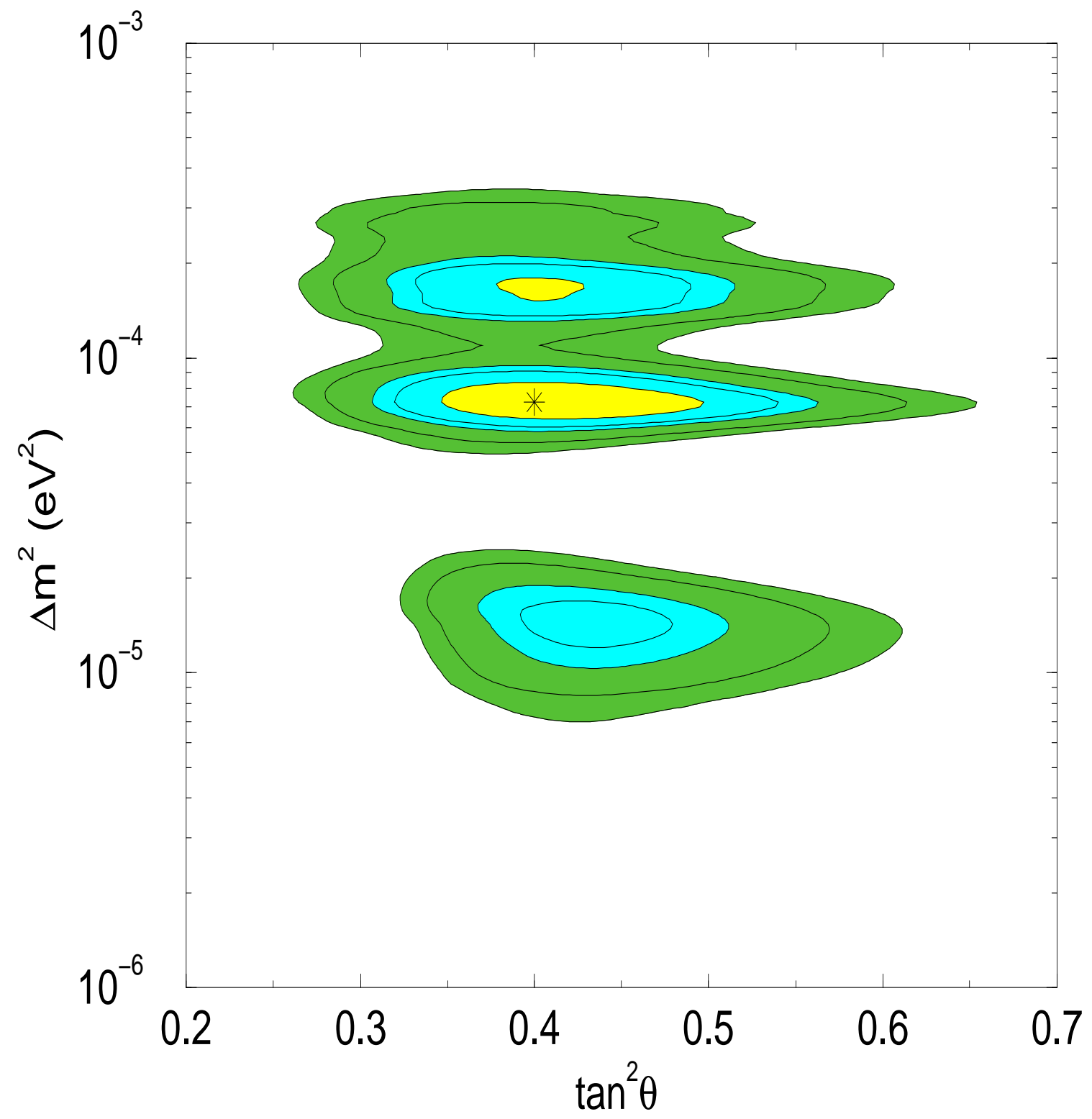

FIG. 2: Allowed regions of oscillation parameters for $1 \sigma, 90 \%$ C.L., 95\% C.L., 99\% C. L. and $3 \sigma$, using the constraints of solar+KamLAND data, with NSNI with d-quarks and where we minimized the $\chi^{2}$ with respect to the NSNI parameter $\epsilon^{\prime}$. The best fit point is marked by a star and happens for $\epsilon^{\prime}=0$. 

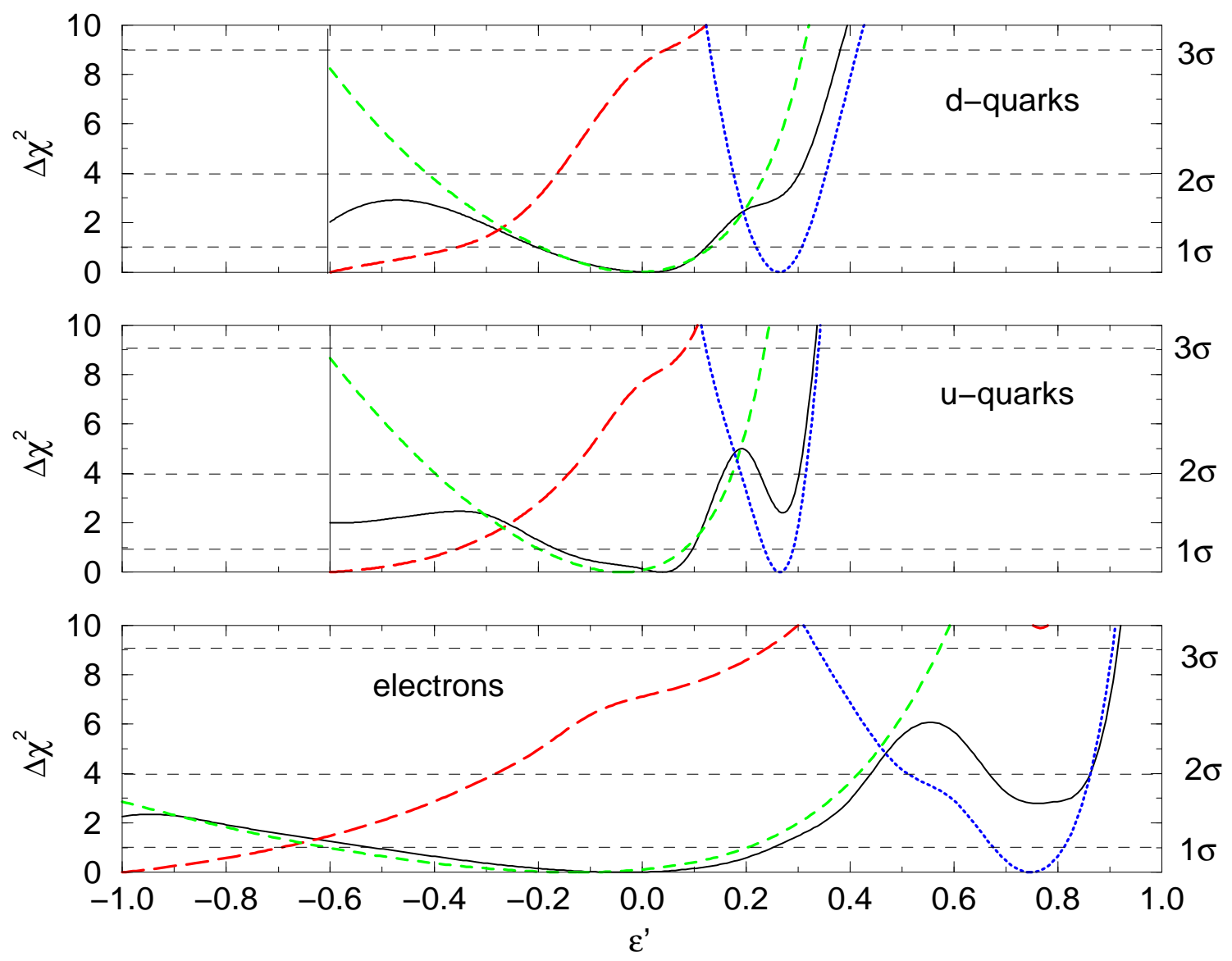

FIG. 3: Future sensitivity of combined analysis of solar+KamLAND, assuming 1kt-yr of exposure. The continuous line corresponds to the actual limit that is obtained with present KamLAND data. The dashed, dotted and long dashed curves refer, respectively, to the simulated parameters $\Delta m^{2}$ and $\tan ^{2} \theta$ lying at low LMA, very low LMA, and high LMA regions. 